\title{
Progress Towards Burning Plasmas ${ }^{*}$
}

\author{
James W. VAN DAM ${ }^{1,2)}$ \\ ${ }^{1)}$ U.S. Burning Plasma Organization $\mathcal{F}$ U.S. ITER Project Office \\ ${ }^{2)}$ Institute for Fusion Studies, The University of Texas at Austin, Austin, Texas 78712, USA
}

(Received 23 September 2008 / Accepted 5 March 2009)

\begin{abstract}
The next frontier for fusion science is the study of burning plasmas. The international ITER facility will advance research efforts into this new regime. In this paper we will first define burning plasmas and describe their distinctive features. One such feature is dominant self-heating (exothermic) by a large population of alpha particles, created from thermonuclear reactions. Next, we will briefly review how previous experiments on JET and TFTR to attain breakeven have laid the foundation for taking the present step to ITER. Then, we will describe various physics and technology issues that need to be addressed for burning plasmas. In addition to the scientific opportunities, we will also describe how ITER, being operated as a large-scale international project, is making progress in terms of organization, mission, funding, and programmatic coordination worldwide.
\end{abstract}

(C) 2009 The Japan Society of Plasma Science and Nuclear Fusion Research

Keywords: burning plasma, fusion energy, thermonuclear reactions, alpha particles, ITER

DOI: $10.1585 /$ pfr.4.035

\section{Introduction}

Understanding the behavior of burning plasmas is the primary challenge faced by fusion research today, as a necessary step towards the ultimate demonstration of fusion as a source of energy [1]. The ITER device, to be operated as an international project, will push research efforts into this new regime of burning plasma science.

Plasmas, sometimes called the "fourth state of matter," span an enormous range of densities and temperatures and include phenomena as diverse as aurora, fluorescent lights and neon signs, interstellar space, the solar wind, lightning, nebula, and - at extremely high temperaturesmagnetic fusion and inertial fusion. Here, we will focus on magnetically confined high-temperature plasmas.

\section{Features of Burning Plasmas}

In burning plasmas, ions undergo thermonuclear fusion reactions, which supply self-heating to the plasma. The well-known binding energy curve, which plots the energy stored in a nucleus versus nuclear mass, shows that the energy output from fusion reactions of low-mass nuclei (e.g., hydrogen) is huge, approximately 450 times the energy input to cause the reaction. This huge output therefore has significant implications for global energy supply. The fusion energy return is much greater than the energy return from fission reactions of heavy nuclei (e.g., uranium). Nevertheless, although the energy return for fusion is large, the required energy input is also large, on the order of $20 \mathrm{keV}$ (equivalent to temperatures of 200 million degrees Kelvin). Hence, fusion is both an opportunity and

author's e-mail:vandam@physics.utexas.edu

*) This article is based on the invited talk at the 14th International Congress on Plasma Physics (ICPP 2008). also a challenge.

Thermonuclear reactions of interest for laboratory fusion power are as follows:

$$
\begin{aligned}
& \mathrm{D}^{+}+\mathrm{D}^{+} \rightarrow{ }^{3} \mathrm{He}^{++}(0.82 \mathrm{MeV})+\mathrm{n}^{0}(2.5 \mathrm{MeV}) \\
& \rightarrow \mathrm{T}^{+}(1 \mathrm{MeV})+\mathrm{p}^{+}(3 \mathrm{MeV}) \\
& \mathrm{D}^{+}+{ }^{3} \mathrm{He}^{++} \rightarrow{ }^{4} \mathrm{He}^{++}(3.6 \mathrm{MeV})+\mathrm{p}^{+}(14.7 \mathrm{MeV}) \\
& \mathrm{D}^{+}+\mathrm{T}^{+} \rightarrow{ }^{4} \mathrm{He}^{++}(3.5 \mathrm{MeV})+\mathrm{n}^{0}(14.1 \mathrm{MeV})
\end{aligned}
$$

In the last of these three reactions, the neutron fusionproduct from the plasma reaction can subsequently be reacted with solid lithium to produce more tritium and another alpha particle (i.e., helium nucleus):

$$
{ }^{6} \mathrm{Li}+\mathrm{n} \rightarrow{ }^{4} \mathrm{He}(2.1 \mathrm{MeV})+\mathrm{T}(2.7 \mathrm{MeV})
$$

The charged alpha particles from fusion reactions can be kept in the plasma by means of magnetic field confinement and thus provide self-heating by giving their large energy to the plasma electrons and ions (mostly the former, which then equilibrate on the latter).

The "easiest" thermonuclear reaction for laboratory fusion uses the hydrogen isotopes of deuterium and tritium (see above). The total energy released per D-T fusion event is $17.6 \mathrm{MeV}$, of which $20 \%$ is carried by the resultant alpha particle and $80 \%$ by a neutron. D-T fusion is easiest for laboratory purposes because the peak value of its nuclear cross section is higher than that for other reactions (e.g., D- $\left.{ }^{3} \mathrm{He}, \mathrm{T}-\mathrm{T}, \mathrm{D}-\mathrm{D}\right)$ and occurs at lower ion energy.

As already indicated, a burning plasma is a plasma that is dominantly self-heated by fusion products (e.g., alpha particles) from thermonuclear reactions in the plasma. This definition can be made more quantitative. Define the fusion energy gain $Q$ as the ratio of the fusion power $P_{\text {fusion }}$ 
to the externally supplied heating power $P_{\text {heat }}$ :

$$
Q=P_{\text {fusion }} / P_{\text {heat }}=5 P_{\alpha} / P_{\text {heat }}
$$

The factor of 5 in the second form of the expression in Eq. (1) applies for D-T fusion, since alpha particles carry $1 / 5$ of the fusion power. Also, define the alpha particle heating fraction $f_{\alpha}=P_{\alpha} /\left(P_{\alpha}+P_{\text {heat }}\right)$ as the fraction of heating power in the alphas. Note that the alpha heating fraction can also be expressed as $f_{\alpha}=Q /(Q+5)$.

"Breakeven" is defined as $Q=1$, which corresponds to an alpha heating fraction of $f_{\alpha}=17 \%$. Initial D-T experiments have already approximately achieved breakeven.

The "burning plasma" regime corresponds to $Q \geq 5$, for which self-heating by alpha particles supplies at least half of the plasma heating (i.e., $f_{\alpha} \geq 50 \%$ ). The international device ITER is designed to achieve $Q=10$ (or $f_{\alpha}=60 \%$ ), and it may be able to study up to $Q=20$ 30. When the alpha particles supply all of the heating $\left(f_{\alpha}=100 \%\right.$ ), external heating is unnecessary, and $Q$ becomes infinite; this corresponds to the "ignition" regime, which is the ultimate target for fusion research.

\section{Science Issues for Burning Plasmas}

In burning plasmas, we will face many of the same scientific issues that are already being addressed on existing experiments. These challenges and associated problem areas include confinement (e.g., $\mathrm{H}$ mode, internal transport barriers, electron thermal transport, momentum transport), MHD macrostability (e.g., resistive wall modes, neoclassical tearing modes, pressure-driven instabilities, edge localized modes, disruptions, sawteeth, fast-ion instabilities), power and particle control (e.g., impurities, plasmafacing component materials, divertor design), long-pulse operation (heating and current drive, profile control, hybrid scenarios), diagnostics (e.g., high temporal and spatial resolution, velocity distribution measurements), and plasma control (start-up, real-time feedback and control). Much progress has been made worldwide in addressing these issues, although in burning plasmas these issues may have new aspects.

The distinctively new scientific challenges to be faced in burning plasmas may be divided into two categories. One such category is related to the new aspects of already known issues when extended to reactor scales in burning plasmas. Here we mention the following issues: (1) those that arise from scaling to large size and strong magnetic field in a fusion reactor; (2) those related to high performance in burning plasmas, with operation near density and beta limits and with very high heat flux on the plasmafacing components; and (3) those related to the nuclear environment of fusion reactor devices, such as radiation, tritium retention, dust, and the need for tritium breeding. A second category of issues are those which are unique to burning plasmas: (1) the existence of a large population of supra-thermal ions, namely, alpha particles; and (2) the fact that burning plasmas will be self-heated, and hence "autonomous" systems with self-organized profiles, as opposed to current devices where the profile can be controlled via externally applied heating and current drive. Related to the self-heating feature of burning plasmas is the issue of thermal stability of the fusion burn cycle.

In addition, the scientific issues in these two categories will all be strongly coupled nonlinearly in burning plasmas. Hence it will be necessary to integrate all of the elements of the scientific challenges in burning plasmas. Incidentally, this highlights the need for integrated numerical simulations for burning plasmas.

A review of the science issues for burning plasmas in ITER may be found in the documents ITER Physics Basis [2] and Progress in the ITER Physics Basis [3] and also in recent workshop presentations [4]. Here, due to space limitations, we focus on three of these issues: alpha particle effects, size scaling, and tritium supply.

As already mentioned, one of the unique features of burning plasmas is the existence of a second population of ions, namely, fusion-product alpha particles, whose properties, however, are quite different from those of the core plasma ions and electrons. Alpha particles are highly energetic: those created from D-T reactions have a birth energy of $3.5 \mathrm{MeV}$, about one hundred times hotter than plasma ions and electrons, whose temperature is typically $10-20 \mathrm{keV}$ in burning plasmas. Due to the large discrepancy in energy, whereas plasma ions and electrons obey the MHD fluid description to lowest order, alpha particles are not "frozen" to the magnetic field lines and therefore require a kinetic description. Plasma ions and electrons are, to lowest order, in thermodynamic equilibrium and have Maxwellian distribution functions in velocity space, whereas alpha particles are non-Maxwellian and instead have a so-called slowing down energy distribution. Furthermore, alpha particles have a centrally peaked pressure profile and can therefore drive universal-type instabilities. For all of these reasons, the behavior of alpha particles is different and must be analyzed accordingly.

In addition to alpha particles, there can be other populations of energetic ions, for example, those produced by neutral beam injection or radio-frequency wave heating. Such energetic ions have been used in current-day experiments to simulate aspects of alpha particle behavior without reactivity. Energetic beam- and rf-generated ions, in addition to alpha particles, will be present in burning plasmas with auxiliary heating. Also, very high-energy electrons, called runaways, can be caused by plasma disruptions. Finally, we note that the physics of supra-thermal ions is of interest not only for laboratory burning plasmas, but has also been applied in space and astrophysical plasmas, an example being the proton ring in the Earth's magnetosphere.

A salient fact is that alpha particles created from D-T reactions can kinetically resonate with shear Alfvén waves because their birth energy $(3.5 \mathrm{MeV})$, when converted into 
velocity, matches the Alfvén speed. Such resonance can cause wave-particle instability. A particularly well-known variety of such instability is the so-called Toroidal Alfvén Eigenmode (TAE), whose theoretical description has a one-to-one analogy to band gaps in solid-state crystals ("fiberglass wave guide"). An entire zoology of other related Alfvén eigenmode instabilities has been elucidated. Of particular concern in burning plasmas is the potential for such instabilities to cause loss of alpha particles, which could lead to reduced self-heating and increased thermal loading on the walls. Much progress, both theoretical and experimental, has been made in characterizing and ameliorating these instabilities and even utilizing their properties to diagnose internal plasma parameters such as magnetic field strength, safety factor, density, and temperature.

The size of burning-plasma experiments is determined by a combination of factors. One such factor is the requirement for sufficient confinement, which is described by scaling predictions for the energy confinement time or the fusion triple product (density times temperature times confinement time). Another factor is the requirement that the materials for the plasma-facing components be able to handle the high power density in burning plasmas. A third factor is the need for adequate radiation shielding of the superconducting magnets; on a scan of the radial build of a burning-plasma device, the vacuum vessel and shield occupy significant space between the plasma and the toroidal field and Ohmic heating coils.

The burning-plasma device ITER will have a cross section four times larger than that of JET, the largest existing tokamak. Its large size means that the normalized Larmor radius $\rho_{\mathrm{i}}{ }^{*}=\rho_{\mathrm{i}} / a$ in ITER will be approximately $10^{-3}$, which is about an order of magnitude smaller than in present-day experiments. New issues arise when this normalized parameter is so small, related to the formation of internal transport barriers, hybrid regimes, confinement scaling, the threshold for neoclassical tearing modes, and the stable spectrum of Alfvén eigenmodes in burning plasmas.

A third science issue for burning plasmas has to do with tritium supply. The D-T fusion reactions consume a large amount of tritium: specifically, $56 \mathrm{~kg}$ of tritium per $1 \mathrm{GW}$ of fusion power per year. Most of the current supply of tritium comes from fission reactors; for example, the CANDU reactors in Canada have produced $27 \mathrm{~kg}$ of tritium over the past 40 years, with the current cost of $\$ 30 \mathrm{M}$ per $\mathrm{kg}$. It has been estimated that the current world supply of tritium would be sufficient for 20 years of ITER operation, which would require about $18 \mathrm{~kg}$. Hence, tritium-breeding technology will be required for the operation of DEMO and other reactors after ITER.

The technology for breeding tritium will be tested on ITER sometime during its research program through the installation of Test Blanket Modules (TBM). ITER has designated three ports for such blanket testing, and two TBMs can be installed in each port. The construction and installation of TBMs is being analyzed so that their ferritic content will not create large magnetic field ripple. In addition to ITER, tritium breeding could be tested by means of fission reactors, accelerator-based point neutron sources, and non-neutron test stands.

\section{Grand Challenge of Burning Plas- mas}

Producing a self-sustaining fusion-heated plasma is indeed a "grand challenge." The history of this endeavor goes back to the beginning of the 20th Century, when Atkinson and Houtermans in 1928 proposed that fusion reactions could explain the energy radiated by stars. In 1932, Oliphant observed fusion reactions in the laboratory. In 1935 Gamow discovered that fusion reactions may be understood as Coulomb barrier tunneling. In 1939 Bethe developed theory for fusion as the power cycle of the stars, for which work he received the Nobel Prize in 1967. During the 1950s, fusion went through a dark period, during which it was used for military objectives. However, during the same period, the tokamak, the stellarator, and the mirror confinement devices were invented. Magnetic fusion research was de-classified internationally at the 2nd United Nations Atoms for Peace Conference in Geneva in 1958. Ten years later, at the 1968 IAEA Plasma Physics and Controlled Fusion Conference held in Novosibirsk, the remarkable Russian results on high-temperature tokamak plasmas were presented. Since then, huge progress worldwide has been achieved in toroidal plasma research, leading to the attainment of fusion-grade plasma parameters.

Initial experiments with deuterium and tritium were carried out during the 1990s on the Joint European Torus (JET) in Europe and the Tokamak Fusion Test Reactor (TFTR) in the US. In 1991, JET performed a "Preliminary Tritium Experiment," which attained fusion power of slightly more than 1 MW. Subsequently, in 1997, JET was able to attain a maximum of $16 \mathrm{MW}$. In terms of fusion performance, JET attained $Q=0.2$ in a long-pulse plasma and $Q=0.9$ (near-breakeven) in a transient discharge. Meanwhile, TFTR operated with 50/50 D-T plasmas from December 1993 until April 1997, running 1,000 discharges, and achieving $P_{\mathrm{DT}}=10.7 \mathrm{MW}$ and $Q=0.2$ (long pulse). The D-T experiments on TFTR were able to observe a number of tritium-related results, including favorable isotope scaling, alpha-particle heating, alphaparticle instability, tritium and helium "ash" transport, and tritium retention in walls and dust. Moreover, TFTR safely handled $1 \mathrm{M}$ curies of tritium, with rigorous accounting. A review of the D-T results from TFTR has been published by Hawryluk [5].

Two paths are being followed in the approach to ignition. One is the high-density path, for which highmagnetic-field proposed tokamaks such as Ignitor, CIT, and FIRE are well suited. The Large Helical Device heliotron has also proposed a high-density approach to igni- 
tion. Another is the high-temperature path, which has been used by TFTR, JET, and JT-60U (Japan) and which will also be used by ITER. Progress in magnetic fusion may be summarized briefly as follows: The high-density path has achieved the ion temperature needed for fusion, with about an order of magnitude increase in the product of density and energy confinement time still required. The hightemperature path, on the other hand, has achieved about half of the value required for the product of density and energy confinement time, and still needs another factor of ten increase in the temperature.

In general, the world fusion program is technically and scientifically ready to proceed now with a burning plasma experiment. Such an experiment is the next logical step forward on the path to fusion energy. For magnetic confinement, ITER is that next step forward.

\section{International ITER Project}

ITER is the essential next step in the development of fusion, since it will demonstrate scientific and technological feasibility. Compared to existing experimental facilities that achieve $10 \mathrm{MW}$ (th) for a few seconds with fusion gain $Q \leq 1$, ITER is designed to achieve $500 \mathrm{MW}$ (th) for discharges of $\geq 400 \mathrm{sec}$ with $Q \geq 10$. When built, it will be the world's largest fusion energy research project, a true "burning plasma" device. It will have a $6.2 \mathrm{~m}$ major radius, a $2.0 \mathrm{~m}$ minor radius, and an $840 \mathrm{~m}^{3}$ plasma volume. It will cost approximately 10B Euros to be constructed, and after first plasma (projected for 2018) it will operate for 20 years. The ITER project is a joint collaboration of seven international partners-China, Europe, India, Japan, Korea, Russian, and the USA - representing more than half of the world's population. The host partner is the European Union, with the actual site in France.

The design goals of ITER encompass both physics and technology. The physics objectives are to produce a plasma dominated by alpha-particle heating, produce a significant fusion power amplification factor $(Q \geq 10)$ in long-pulse operation, aim to achieve steady-state operation of a tokamak $(Q=5)$, and retain the possibility of exploring "controlled ignition" ( $Q \geq 30)$. The technology objectives are to demonstrate integrated operation of technologies for a fusion power plant, test components for a fusion power plant, and test concepts for a tritium breeding module.

Even before the ITER project began, there was a multi-national effort called the International Tokamak Reactor (INTOR) Workshop, which lasted from 1978 to 1981. Sponsored by the International Atomic Energy Agency, it involved four partners (Euratom, Japan, USA, and USSR). Its final product was a conceptual design for a $600 \mathrm{MW}(\mathrm{th})$ device, described in an accompanying 860-page report.

ITER itself began with a Conceptual Design Activity (CDA) phase, from 1987 to 1990. An Engineering Design Activity phase lasted from 1992 to 1998, with four partners (EU, JA, RF, and US). For the EDA phase, ITER had joint work sites in Garching (EU), Naka (JA), and San Diego (US). This phase was succeeded by the ITER Fusion Ignition Advanced Tokamak (FIAT) effort during 1999 to 2003. For various reasons, the US withdrew from the ITER Project in 1998 and re-entered in 2003. On November 21, 2006, the ITER Implementing Agreement was signed by all of its partners, and in October 2007 the ITER Organization was established as a legal entity. With the addition of India in 2005, there are now seven partners in the ITER enterprise.

Through international negotiations, it was eventually determined that Europe would be the host partner, with the ITER laboratory to be situated next to the CEA nuclear research center in Cadarache, France, about an hour by car north of Marseille.

The funding arrangements for ITER are such that the host partner (EU) will contribute 5/11ths of the construction cost, with each of the other six partners providing $1 / 11$ th in in-kind contributions. In January 2008, Monaco signed a partnership arrangement with ITER in which it will provide funds to support ITER postdoctoral fellowships and host international conferences. Recently, Kazakhstan has expressed interest in becoming a full partner in ITER.

The organization structure for ITER has been carefully thought out. ITER is governed by a Council, with high-level representatives from the seven partners. The Council has a Management Advisory Committee (MAC) and a Science and Technology Advisory Committee (STAC). The ITER Organization itself has a DirectorGeneral and seven Deputy Director-Generals, one of whom, as the Principal Deputy Director-General, is also the Project Construction Leader. The Director-General, Mr. Kaname Ikeda, and the Principal Deputy DirectorGeneral, Dr. Norbert Holtkamp, have both had distinguished careers in the management of large scientific projects. More information about ITER is available on its web site [6].

Within the US, the efforts in burning plasma science are coordinated through several structures, beginning with the Office of Fusion Energy Sciences in the US Department of Energy. To the US ITER Project Office, hosted at Oak Ridge National Laboratory, has been assigned the responsibility as the official US Domestic Agency for ITER. The US ITER Project Office, headed by Dr. Ned Sauthoff, oversees procurement arrangements etc. for the US share of the construction of ITER. The US ITER Project Office has a chief scientist, who is also director of the US Burning Plasma Organization (USBPO), which promotes, coordinates, and facilitates burning-plasma physics research. On the national level, the USBPO is integrated with the International Tokamak Physics Activity (ITPA). The US ITER Project Office also has a chief technologist, who is also director of the US Virtual Laboratory for Technology. More information about these offices and organizations can be obtained from their respective web sites [7]. 
A large, international project like ITER has challenges that extend beyond those related to scientific issues. For example, in terms of communications, ITER strives to employ modern video-conferencing techniques and integrated document management. ITER must also deal with such issues as intellectual property rights to data, cultural differences and management styles, multi-national safety regulations, import and export regulations, and public outreach for education and visibility. The US ITER Project Office produced a short three-dimensional movie about ITER, which it exhibited at the 2008 American Association for the Advancement of Science Annual Meeting; a clip about ITER wave heating from this movie can be found at the YouTube web site.

\section{Conclusion}

Since the time of the 2nd UN Conference on Peaceful Uses of Atomic Energy (1958), the worldwide fusion energy effort has made great scientific and technical progress. This progress has been facilitated by an emphasis on international collaborations and motivated by an awareness of the potential benefit of fusion energy for all humanity.

The next frontier for fusion science is the study of burning plasmas. The ITER project—an unprecedented model for big-science international collaboration-will advance the development of fusion into this exciting new regime.

\section{Acknowledgments}

Support is acknowledged from the Office of Fusion Energy Sciences of the U.S. Department of Energy and from the U.S. Burning Plasma Organization and the U.S. ITER Project Office.

[1] J. Ahearne, R.J. Fonck and Burning Plasma Assessment Committee, Burning Plasma: Bringing A Star To Earth (US National Academy of Science, 2004).

[2] ITER Physics Basis, Nucl. Fusion 39 (12), 2137 (1999).

[3] Progress in the ITER Physics Basis, Nucl. Fusion 47 (6), S1 (2007).

[4] See presentations given at the USBPO Burning Plasma Workshop (www.burningplasma.org/reference.html).

[5] R. Hawryluk, Rev. Mod. Phys. 70, 537 (1998).

[6] See web site for ITER Organization (www.iter.org).

[7] See web sites for USDOE Office of Fusion Energy Sciences (http://www.science.doe.gov/ofes/), US ITER Project Office (www.usiter.org), US Burning Plasma Organization (www.burningplasma.org), and US Virtual Laboratory for Technology (http://www.ornl.gov/sci/vlt/). 